\title{
QUALIDADE DA MADEIRA PARA PRODUÇÃO DE LÂMINAS DE Araucaria angustifolia (Bert.) O. Kuntze
}

\author{
Ana Carolina Caixeta Dias ${ }^{1 *}$, Raquel Marchesan ${ }^{2}$, Gustavo Bloise Pieroni ${ }^{3}$, Vanessa Coelho Almeida ${ }^{2}$, \\ Renato Silva Vieira ${ }^{4}$, Cristiano Bueno de Moraes ${ }^{2}$ \\ ${ }^{1 *}$ Universidade Federal do Tocantins, Programa de Pós-Graduação em Ciências Florestais e Ambientais, Gurupi, Tocantins, Brasil - \\ vania.wcaixeta@gmail.com \\ ${ }^{2}$ Universidade Federal do Tocantins, Curso de Engenharia Florestal, Gurupi, Tocantins, Brasil - raquelmarchesan@uft.edu.br*; \\ vanessacoelhoalmeida@uft.edu.br; cbmoraes@uft.edu.br \\ ${ }^{3}$ Empresa PalmaSola S/A, Palma Sola, Santa Catarina, Brasil - gustavo@palmasola.com.br \\ ${ }^{4}$ Universidade Federal de São João del-Rei, Curso de Engenharia Florestal, São João del-Rei, Minas Gerais, Brasil - \\ renatovieira@ufsj.edu.br
}

Recebido para publicação: 17/02/2017- Aceito para publicação: 25/09/2017

\begin{abstract}
Resumo
O objetivo deste estudo foi avaliar a qualidade de toras e madeira de Araucaria angustifolia para o processo de laminação destinado a produção de painéis laminados. As árvores foram provenientes de um plantio seminal de 39 anos de idade, localizado no município de Palma Sola, SC. A qualidade das toras para laminação foi analisada quanto a conicidade e o achatamento de acordo com IBDF (1984). O colapso da madeira foi determinado seguindo a metodologia adotada por Welling (1994) e a densidade básica e retração obedeceram à norma NBR 7190 (ABNT, 1997) e NBR 11941 (ABNT, 2003) respectivamente. Os resultados encontrados para as características de forma do fuste apresentam-se com características favoráveis a produção de lâminas homogêneas, de acordo com a classificação do IBDF (1984). A densidade básica na direção radial apresentou aumento significativo da medula à casca, enquanto no sentido longitudinal, foi decrescente da base ao topo, com tendência de estabilização dos valores a partir da posição referente a $75 \%$ da altura comercial. O colapso da madeira foi caracterizado como de grau médio e a variação dimensional classificada como de alta estabilidade, podendo ser utilizadas para a produção de painéis laminados.
\end{abstract}

Palavras-chave: Densidade básica; Retratibilidade; Colapso; Achatamento.

\begin{abstract}
Wood quality for Araucaria angustifolia (Bert.) O. Kuntze laminated wood production. The objective of this study was to evaluate the quality of Araucaria angustifolia logs and wood for the production of laminated panels. The trees came from a seminal planting of 39 years old, located in the city of Palma Sola, SC. The quality of the logs for lamination was analyzed for conicity and flatness according to IBDF (1984). The collapse of the wood was determined following the methodology adopted by Welling (1994) and the basic density and shrinkage obeyed the standard NBR 7190 (ABNT, 1997) and NBR 11941 (ABNT, 2003) respectively. The results obtained for the shape characteristics of the stem form are presented with favorable characteristics to the production of homogeneous veneer, according to the IBDF classification, (1984). The variation of the basic density in the radial direction presented significant increase of the marrow to the bark, while in the longitudinal direction, it was decreasing from the base to the top, with the basic density average at $75 \%$ of the commercial height. The wood collapse was presented as medium grade and the dimensional variation classified as high stability, and can be used for the production of laminated panels.

Keywords: Basic density; shrinkage; Collapse; Flattening.
\end{abstract}

\section{INTRODUÇÃO}

A Araucaria angustifolia é uma espécie importante no Brasil por apresentar características favoráveis para a indústria de base florestal. A espécie passou por processo de devastação de forma inconsciente a partir de 1910 para construção de rodovias e fabricação de painéis compensados, desta forma é considerada uma espécie ameaçada de extinção (IBAMA, 1992). Para suprir a demanda madeireira, plantios comerciais com a espécie na região Sul do Brasil foram intensificados com o surgimento dos incentivos fiscais no ano de 1966. A partir de então, empresas e instituições de pesquisa têm investido nestes plantios para melhorar qualidade da madeira. Segundo a ABRAF (2013) a área de florestas plantadas de araucária abrange 11.343 hectares no país.

FLORESTA, Curitiba, PR, v. 47, n. 3, p. 333 - 341, jul. / set. 2017.

Dias, A. C. C. et al.

ISSN eletrônico 1982-4688

DOI: $10.5380 /$ rf.v47i1.50732 
Segundo Figueiredo Filho et al. (2015) a principal fonte de madeira de araucária disponível no mercado decorre de florestas plantadas. Desta forma, é essencial investir para viabilizar o plantio comercial em larga escala, tendo em vista a sua importância econômica, ambiental e cultural (SANQUETTA et al., 2016).

As indústrias madeireiras foram atraídas pela espécie por proporcionar uma menor geração de resíduos no processamento quando comparada com espécies mais usuais como Pinus e Eucalyptus (VALÉRIO et al., 2007) e em razão das características favoráveis à laminação, destacando a baixa conicidade, estabilidade dimensional e densidade básica. Essa última tem sido considerada como o principal índice de qualidade da madeira por influenciar as demais propriedades além de ser de fácil determinação.

Apesar de a densidade básica ser o principal parâmetro indicativo de qualidade, não assegura a qualidade da árvore selecionada no intuito de fornecer matéria-prima adequada para produção de lâminas. A definição de outras características, tais como a forma do fuste e outras propriedades físicas como retratibilidade e colapso, tornam-se de grande importância para atender a demanda das indústrias de laminação com material de qualidade.

A madeira considerada de melhor qualidade é aquela que apresenta menor quantidade de defeitos, como a formação de nós, veios de quino, além da conicidade e achatamento, podendo ser intrínsecos (genéticos) ou resultantes do processo de corte, desdobro e/ou secagem da madeira. Medidas relativas aos tratos silviculturais no povoamento e métodos de secagem procuram amenizar os defeitos para a produção de lâminas, tanto na formação dos painéis como no processamento e acabamento. Por se tratar de material heterogêneo inter e intraespecífico, requerem estudos aprofundados visando aumentar a produtividade da madeira para produção de lâminas com qualidade (AMORIN et al., 2013). A escassez de trabalhos relacionados à conicidade e principalmente ao achatamento da madeira de araucária reforça a importância de se realizar trabalhos nesta área a fim de adequar técnicas que possam atenuar tais defeitos e gerar melhor aproveitamento da madeira.

Neste contexto, torna-se necessário estudar a qualidade de sua madeira procedente de plantios comerciais por meio da avaliação da qualidade do fuste e da madeira, para avaliar seu potencial madeireiro quanto à qualidade de sua madeira para produção de painéis.

\section{MATERIAL E MÉTODOS}

\section{Material e localização geográfica}

Foram utilizadas 25 árvores matrizes de Araucaria angustifolia de 39 anos de idade provenientes de um plantio seminal comercial da empresa Palmasola S/A, obtidas de único talhão contendo 30 ha. A área localiza-se no Município de Palma Sola, Santa Catarina (latitude 26²0' S, longitude 53ำ $16^{\prime} \mathrm{W}$ e altitude aproximada de 870 metros). O solo do local é classificado como Latossolo Bruno Distrófico de textura argilosa.

\section{Características morfométricas}

Para avaliação das características morfométricas foram considerados os parâmetros conicidade e achatamento em fustes de madeira de 20 metros de comprimento. Cada defeito foi medido e classificado conforme a Norma para medição e classificação de toras de madeiras IBDF (1984).

A conicidade $(\mathrm{cm} / \mathrm{m})$ foi determinada pela diferença entre o diâmetro da base e o diâmetro do topo da tora dividido pelo comprimento da mesma. $\mathrm{O}$ achatamento das toras foi determinado por meio de medições dos diâmetros da base e do topo, tomando-se duas medidas em cada extremidade e calculando-se a média destas medidas.

$$
C(\%)=\frac{\frac{\mathrm{d} 1+\mathrm{d} 2}{2}-\frac{\mathrm{d} 3+\mathrm{d} 4}{2}}{\mathrm{Lt}}
$$

em que: $\mathrm{C}(\%)$ : Conicidade; d: diâmetros da base e topo; $\mathrm{Lt}$ : comprimento da tora.

$$
\mathrm{A}(\%)=\frac{\frac{\mathrm{d} 1+\mathrm{d} 2}{2}}{\frac{\mathrm{d} 3+\mathrm{d} 4}{2}} * 100
$$

em que: A(\%): Achatamento; d: diâmetros da base e do topo. 


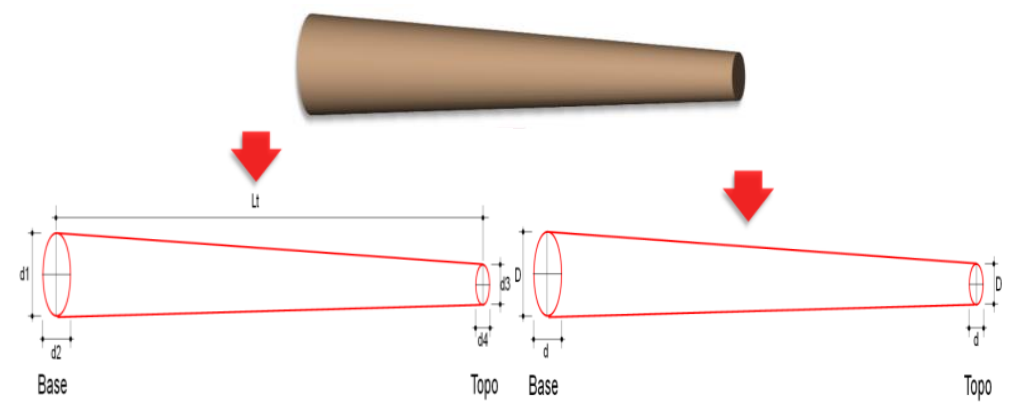

Figura 1. Esquema representativo para cálculo do colapso e achatamento.

Figure 1. Representative scheme for calculating collapse and flattening.

\section{Propriedades físicas da madeira}

Determinação da densidade básica

A densidade básica da madeira foi determinada de acordo com a norma NBR 11941 (ABNT, 2003). Para a variação na direção longitudinal, utilizaram-se cinco discos por árvore, sendo extraídos nas alturas de $0 \%$, $25 \%, 50 \%, 75 \%$ e $100 \%$ da altura comercial a partir de cunhas de madeira. Para determinar a variação radial, utilizou-se um disco retirado a 1,3 metros do solo (DAP) a partir de um sarrafo do meio do disco, em que foi obtido o mesmo número de amostras de cada lado em três posições no sentido medula casca: próximo à medula, intermediário e próximo à casca, com as dimensões adotadas conforme a NBR 7190 (ABNT, 1997), 200 x 300 x $500 \mathrm{~mm}$ nas direções lineares radiais, tangenciais e longitudinais, respectivamente.

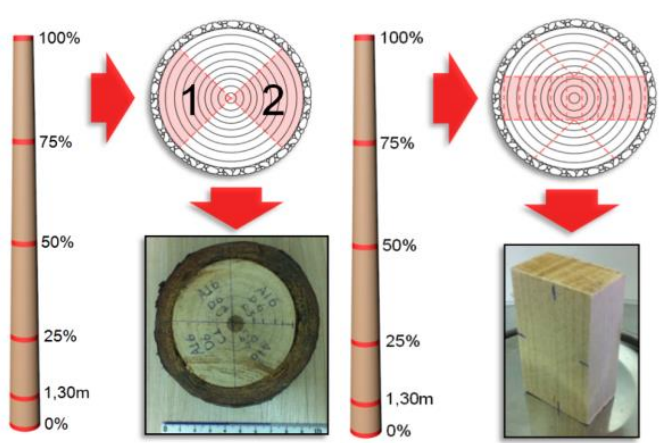

Figura 2. Esquema representativo da coleta dos discos e corpos de prova na direção longitudinal e radial, respectivamente (Fonte: A autora).

Figure 2. Representative scheme of the collection of the test discs and specimens in the longitudinal and radial directions, respectively (Source: The author).

\section{Colapso}

A determinação do colapso seguiu a metodologia adotada por Welling (1994) conforme a tabela 1. Foram realizadas medições nos eixos lineares por meio da deformação das áreas de maior e menor espessura nas condições de volume verde e massa seca em todos os corpos de prova.

Tabela 1. Classificação do grau de colapso de madeira. Adaptado de Welling (1994).

Table 1. Classification of the collapse of wood grade. Adapted from Welling (1994).

\begin{tabular}{l|l}
\hline Grau do colapso & Redução na espessura \\
\hline Baixo & 0 a $2 \mathrm{~mm}$ \\
Moderado & 2 a $4 \mathrm{~mm}$ \\
Forte & 4 a $6 \mathrm{~mm}$ \\
\hline
\end{tabular}

\section{Retratibilidade e Coeficiente de anisotropia}

As mesmas amostras utilizadas na determinação da densidade básica foram empregadas na avaliação da retração radial, tangencial, longitudinal e volumétrica, obedecendo à norma NBR 7190 (1997). 


\section{Análise dos dados}

Empregou-se, para a análise, um delineamento inteiramente casualizado (DIC) em que foram realizados testes de normalidade (Shapiro-Wilk) e o teste de homogeneidade (Bartlett), para realizar a análise estatística paramétrica a partir das análises de variância (ANOVA). Quando a hipótese de nulidade da ANOVA foi rejeitada foi realizada a análise não paramétrica utilizando o teste Tukey. Também foi determinado o coeficiente de correlação de Pearson (r) para verificar a relação entre a densidade básica e as demais características avaliadas.

\section{RESULTADOS}

\section{Propriedades morfométricas}

Na tabela 2 constam os valores de conicidade e achatamento para a espécie de Araucaria angustifolia.

Tabela 2. Valores de conicidade e achatamento para avaliar a qualidade da madeira de Araucaria angustifolia.

Table 2. Conicity and flatness values to evaluate the quality of Araucaria angustifolia wood.

\begin{tabular}{ccc}
\hline & Conicidade (C\%) & Achatamento (A\%) \\
\hline Mínimo & 0,40 & 88,29 \\
Médio & 0,52 & 95,08 \\
Máximo & 0,65 & 99,07 \\
\hline DP & 0,06 & 3,06 \\
\hline CV & 12,11 & 3,22 \\
\hline Nota: DP (desvio padrão); CV (coeficiente de variação). \\
\hline
\end{tabular}

\section{Propriedades físicas}

Variação longitudinal da densidade básica

Na tabela 3, são apresentados os dados médios de densidade básica obtidos a partir dos discos retirados nas alturas de $0,25,50,75$ e $100 \%$.

Tabela 3. Variação da densidade básica no sentido longitudinal expressa em g.cm- ${ }^{3}$ em árvores de Araucaria angustifolia.

Table 3. Variation of the basic density in the longitudinal direction expressed in g.cm- ${ }^{3}$ in Araucaria angustifolia trees.

\begin{tabular}{ccc}
\hline Tratamento & DB $\left(\mathbf{g . c m} \mathbf{-}^{\mathbf{3}}\right)$ & CV (\%) \\
\hline $100 \%$ & $0,34 \mathrm{a}$ & $(7,65)$ \\
$75 \%$ & $0,36 \mathrm{ab}$ & $(7,16)$ \\
$50 \%$ & $0,38 \mathrm{~b}$ & $(6,63)$ \\
$25 \%$ & $0,42 \mathrm{c}$ & $(8,59)$ \\
$0 \%$ & $0,46 \mathrm{~d}$ & $(6,38)$ \\
\hline $\mathrm{DB}_{\mathrm{m}}$ & 0,41 & \\
\hline $\mathrm{F}$ & $64,26 * *$ & \\
\hline
\end{tabular}

Nota: $\mathrm{DBm}=$ Densidade básica média ponderada em função do volume. As médias seguidas pela mesma letra não diferem estatisticamente entre si. Teste Tukey ao nível de $5 \%$ de probabilidade. **significativo ao nível de $1 \%$ de probabilidade $(\mathrm{p}<0,001)(\mathrm{p}: 0,007)$. Valores entre parêntese indicam o coeficiente de variação. CV (coeficiente de variação).

\section{Densidade básica no sentido radial}

Na tabela 4 consta a variação radial da densidade básica da madeira.

Tabela 4. Variação da densidade básica no sentido radial expressa em ${\mathrm{g} . \mathrm{cm}^{-3}}^{\mathrm{em}}$ árvores de Araucaria angustifolia.

Table 4. Variation of the basic density in the radial direction expressed in ${\mathrm{g} . \mathrm{cm}^{-3}}^{-}$in Araucaria angustifolia trees.

\begin{tabular}{ccc}
\hline Tratamento & DB $\left(\mathbf{g . c m}^{-\mathbf{3}}\right)$ & $\mathbf{C V}$ \\
\hline Próximoà medula & $0,36 \mathrm{c}$ & $(7,01)$ \\
Intermediário & $0,42 \mathrm{~b}$ & $(6,33)$ \\
Próximo à casca & $0,45 \mathrm{a}$ & $(5,5)$ \\
\hline Média & 0,41 & $(6,93)$ \\
\hline $\mathrm{F}$ & $59,61^{* *}$ &
\end{tabular}

Nota: As médias seguidas pela mesma letra não diferem estatisticamente entre si. Teste Tukey ao nível de 5\% de probabilidade.** significativo ao nível de $1 \%$ de probabilidade $(\mathrm{p}<01)$. (p: 0,001$)$ Valores entre parêntese indicam o coeficiente de variação. 
Colapso e retrações da madeira

$\mathrm{Na}$ tabela 5, apresentam-se os resultados de colapso, retrações e coeficiente de anisotropia da madeira de araucária.

Tabela 5. Parâmetros físicos avaliados para a madeira de Araucaria angustifolia.

Table 5. Physical parameters evaluated for Araucaria angustifolia wood.

\begin{tabular}{lccccc}
\hline \multirow{2}{*}{ Tratamento } & \multirow{2}{*}{ Colapso $(\mathbf{m m})$} & \multicolumn{3}{c}{ Retração } & \multirow{2}{*}{ CA (\%) } \\
\cline { 3 - 5 } Próximo à medula & $2,6912 \mathrm{~b}$ & 4,2176 & $\mathbf{R d}(\boldsymbol{\%})$ & vol (\%) & \\
& $(12,09)$ & $(16,55)$ & $(15,4596$ & 7,0352 & 1,5440 \\
Intermediário & $3,0060 \mathrm{a}$ & 4,6268 & 3,1668 & $7,01)$ & $(11,38)$ \\
& $(8,20)$ & $(15,08)$ & $(17,07)$ & $(13,79)$ & $(10,4680$ \\
Próximo à casca & $2,8720 \mathrm{ab}$ & 4,3088 & 3,0504 & 7,3508 & 1,4616 \\
& $(18,48)$ & $(20,11)$ & $(26,27)$ & $(20,11)$ & $(19,50)$ \\
\hline Média & 2,8564 & 4,3844 & 2,9929 & 7,401 & 1,4912 \\
& $(13.54)$ & $(17,24)$ & $(20,29)$ & $(16,18)$ & $(14.31)$ \\
\hline $\mathrm{F}$ & $4,16^{*}$ & $2,020 \mathrm{~ns}$ & $3,006 \mathrm{~ns}$ & $2,697 \mathrm{~ns}$ & $1,1535 \mathrm{~ns}$ \\
\hline
\end{tabular}

Nota: Retração tangencial (tg), retração radial (rd), retração volumétrica (vol) coeficiente de anisotropia (CA).As médias seguidas pela mesma letra não diferem estatisticamente entre si. Teste Tukey ao nível de 5\% de probabilidade.* significativo ao nível de 5\% de probabilidade **significativo ao nível de $1 \%$ de probabilidade $(\mathrm{p}<01)$. Valores entre parêntese indicam o coeficiente de variação.

\section{Correlação de Pearson}

A tabela 6 apresenta o coeficiente de correlação (r) obtido entre a densidade básica e os parâmetros físicos avaliados para a madeira de Araucaria angustifolia.

Tabela 6. Coeficiente de correlação de Pearson (r) entre a densidade básica e parâmetros avaliados para a madeira de Araucaria angustifolia.

Table 6. Pearson correlation coefficient (r) between basic density and evaluated parameters for Araucaria angustifolia wood.

\begin{tabular}{cccccc}
\hline \multirow{2}{*}{ Posições } & \multirow{2}{*}{ Colapso } & \multicolumn{3}{c}{ Retração } & Ca \\
\cline { 3 - 5 } & & Tg & Rd & 0,283 & 0,238 \\
Próximo à medula & 0,191 & 0,232 & 0,371 & 0,265 \\
Intermediária & 0,178 & 0,376 & 0,160 & 0,278 \\
Próximo à casca & 0,277 & 0,044 & 0,220 & 0,111 \\
\hline Nota: Retração tangencial $(T g)$, retrac̃ão radial (Rd), retração volumétrica (Vol) coeficiente de anisotropia $(\mathrm{Ca})$.
\end{tabular}

Nota: Retração tangencial (Tg), retração radial (Rd), retração volumétrica (Vol) coeficiente de anisotropia (Ca).

\section{DISCUSSÃO}

\section{Propriedades morfométricas}

Segundo IBDF (1984) para um fuste ser caracterizado como defeito (cônico), deve estar acima de 3,0\%. De acordo com Reitz e Klein (1966) o fuste de araucária é bastante cilíndrico, o que foi verificado na analise da tabela 2, com os valores abaixo do considerado defeito, averiguando a baixa conicidade da espécie.

Este defeito pode estar associado a características peculiares da espécie, idade e influências ambientais. Em casos de plantios com espaçamento reduzido, normalmente, apresentam forma mais cilíndrica, como verificado neste estudo. Árvores com baixo grau de conicidade são indicadas para o uso na laminação devido à maior homogeneidade do diâmetro ao longo do fuste e da densidade básica. A baixa variação do diâmetro pode gerar lâminas de melhor qualidade resultante de árvores inteiras, além do maior aproveitamento do fuste com menor geração de resíduos no processo de faqueamento ou torneamento, enquanto a homogeneidade da densidade básica é recorrente da disposição das células juvenis e adultas ao longo do fuste, o que justifica a ampla utilização da espécie para laminação.

Ao relacionar com a idade das árvores Figueredo Filho et al. (2014) estabeleceram uma correlação com a forma do tronco da araucária e observaram que a conicidade tende a diminuir com a idade. Até os 4 anos, a conicidade é normalmente maior que 1, tendendo a se estabilizar a partir dos 35 anos de idade com fator de conicidade em torno de 0,6 . Estes autores verificaram que em plantios em sítios mais produtivos de araucária, a partir dos 35 anos de idade, as árvores apresentam formato cilíndrico recomendado para produção de lâminas, e somente a partir de 50 anos para as árvores em sítios de menor produção.

Ainda de acordo com a tabela 2, o valor médio do fator achatamento, foi considerado satisfatório para este estudo considerando os limites de tolerância estipulados pela norma IBDF (1984), sendo considerado defeito

FLORESTA, Curitiba, PR, v. 47, n. 3, p. 333 - 341, jul. / set. 2017

Dias, A. C. C. et al.

ISSN eletrônico 1982-4688

DOI: $10.5380 /$ rf.v47i1.50732 
quando apresentar-se abaixo de $90 \%$. Em se tratando de madeiras destinadas à laminação, árvores que tem um achatamento considerado alto apresentam o crescimento excêntrico da medula e a presença do lenho de compressão, levando a obstrução das paredes celulares. Como consequência, as lâminas ficam propensas a alta instabilidade dimensional e menor resistência mecânica. Para madeiras que apresentam lenho de compressão, as lâminas podem apresentar distorções sendo mais propensas ao aparecimento de trincas, também recorrentes da menor resistência da madeira.

\section{Propriedades físicas}

Variação da densidade básica no sentido longitudinal

A variação no sentido base-topo apresentou comportamento decrescente significativo da base $\left(0,46 \mathrm{~g} \cdot \mathrm{cm}^{-3}\right)$ para o topo $\left(0,34 \mathrm{~g} \cdot \mathrm{cm}^{-3}\right)$, portanto, com uma tendência da densidade diminuir conforme o aumento da altura das árvores.

A menor densidade básica encontra-se no topo das árvores uma vez que a parede celular da madeira juvenil apresenta lumes grandes e parede celular menos espessa quando comparada com madeira jovem. Este comportamento esta associado à maior proporção de madeira adulta nas porções basais do fuste com tendência a diminuir conforme a altura. Além da proporção de madeira juvenil, os anéis de crescimento também influenciam fortemente na baixa densidade básica. Mattos (2011) obtiveram para a araucária com 19 anos de idade, densidades de 0,$47 ; 0,45 ; 0,42 ; 0,39 ; 0,36$ e $0,33{\mathrm{~g} . \mathrm{cm}^{-3}}^{-3}$ para alturas no DAP e $0,25,50,75$ e $100 \%$ respectivamente, diferindo estatisticamente entre as alturas.

A densidade encontrada na altura a $75 \%$ não apresentou diferença significativa em relação às densidades a $50 \%$ e $100 \%$, demonstrando uma tendência de se tornar mais constante próximo ao topo. Curto et al. (2016) em pesquisa realizada com a espécie A. angustifolia com 65 anos observou que a posição a $60 \%$ apresentou maior densidade em relação a de $40 \%$ demonstrando seu indicativo de efeito de competição na área, ocasionado por falta de manejo.

\section{Variação da densidade básica no sentido radial}

O Instituto Brasileiro do Meio Ambiente e dos Recursos Naturais Renováveis (IBAMA) (2015) classifica as madeiras conforme suas densidades como leves $\left(<0,50 \mathrm{~g} . \mathrm{cm}^{-3}\right)$, moderadas (entre 0,51 e $\left.0,72 \mathrm{~g} \cdot \mathrm{cm}^{-3}\right)$ e pesadas $\left(>0,73 \mathrm{~g} \cdot \mathrm{cm}^{-3}\right)$.

A variação da densidade básica da madeira $\left(0,36\right.$ a $\left.0,45 \mathrm{~g} \cdot \mathrm{cm}^{-3}\right)$ é compatível com os resultados encontrados por outros autores como Mattos et al. (2006) e Curto et al. (2016) em se tratando da espécie $A$. angustifolia proveniente de plantios florestais. Esta madeira se enquadra na classificação de madeiras leves, onde não requer resistência mecânica, utilizadas basicamente na produção de lâminas para a fabricação de compensados e aglomerados de diversos tipos.

Mattos et al. (2006) estudando a araucária com 38 anos de idade, obtiveram média de densidade básica de $0,42 \mathrm{~g} . \mathrm{cm}^{-3}$, valor semelhante ao encontrado neste estudo. Entretanto, Curto et al. (2016) avaliando a densidade básica em araucária com 65 anos na altura do DAP, encontraram resultados acima do obtido neste estudo, com média da densidade básica de $0,48 \mathrm{~g} \cdot \mathrm{cm}^{-3}$.

Observa-se na tabela 4 diferença estatística significativa da densidade básica aumentando no sentido medula-casca, recorrente da formação das células juvenis e adultas. Nas gimnospermas, quanto mais próxima da medula, a madeira apresenta comportamento que tende a ser mais acentuado, e quanto mais próximo à casca reduz a intensidade de crescimento, tendendo a se tornar constante. A madeira próxima à medula apresenta traqueídes mais finos e menor diâmetro do lume, o que proporciona a menor densidade. Já na região próxima à casca, os anéis de crescimento se dispõem com mais proximidade, resultante de lenho tardio que apresentam como característica maior espessura da parede celular e lumes delgados, resultando, portanto, em maior densidade básica. Wehr e Tomazello Filho (2000) ao estudar araucária notaram que a densidade aumentou da medula à casca, entretanto, não houve diferença estatística entre as posições.

\section{Colapso e retrações da madeira}

Observa-se que a madeira próxima à medula e a intermediária diferiram significativamente entre si a $5 \%$ de probabilidade para o colapso, enquanto que, a madeira próxima à casca não apresentou significância entre as demais. A madeira de araucária apresentou valores de colapso moderados entre 2 e 4 , conforme a classificação de Welling (1994) tendo a madeira de cerne o melhor desempenho.

Este resultado baseia-se na teoria de que a madeira próxima à medula e próxima à casca apresentam diferentes teores de umidade. Apenas as camadas externas do lenho possuem atividades fisiológicas ativas, com fluxo de líquidos no sentido ascendente, saturados por água capilar que pode ser facilmente retirada, excedendo a resistência às forças capilares, aumentando a tendência o colapso. O comportamento é explicado em virtude de 
que o colapso esta diretamente relacionada com a permeabilidade da madeira, a qual é influenciada pela densidade, diâmetro dos capilares, dentre outros fatores.

Klitzke (2007) classificou a estabilidade quanto à retração volumétrica em níveis sendo: alta estabilidade quando apresentar retração inferior a $8 \%$, estabilidade média para retração entre 8 e $10 \%$, baixa estabilidade para aquelas entre 12 e $15 \%$ e instáveis quando acima de $15 \%$. A estabilidade do lenho, em geral, pode ser atribuída à existência de grande parte de lenho adulto, apresentando menor ângulo das microfibrilas e raios mais próximos, proporcionando menor retração. Rosa et al. (2013) verificou que a madeira de araucária obtida próxima à casca apresentou maior contração volumétrica quando comparada com o lenho próximo da medula.

Em relação ao resultado das retrações lineares tangencial e radial médias, as mesmas apresentaram valores considerados baixos quando comparados com os autores Melo et al. (2010) e semelhante ao trabalho realizado por Hillig et al. (2012). Estas diferenças encontradas nas retrações para uma mesma espécie, provavelmente ocorreram ao maior percentual de lenho adulto com paredes espessas, responsável por conferir maior estabilidade, além da diferença anatômica do fuste, proporções de traqueóides, ângulo microfibrilar, da distância entre os anéis de crescimento e da constituição química, influenciadas pela idade da árvore.

Estas diferenças encontradas nas retrações, neste estudo com os demais, provavelmente ocorreu pela diferença anatômica do fuste, proporções de traqueóides, ângulo microfibrilar, da distância entre os anéis de crescimento além da constituição química, influenciadas pela idade da árvore.

Melo et al. (2010) em um trabalho comparando madeira de lenho juvenil e lenho adulto de Araucaria angustifolia com 50 anos de idade, obtiveram retrações radial e tangencial de 5,27 e 7,41 respectivamente para o lenho juvenil. Para o lenho adulto, os resultados apresentados foram de 4,11 e 6,73. Estes autores concluíram que o lenho próximo à casca (lenho adulto) apresentou-se mais estável que o lenho próximo à medula (lenho juvenil). Hillig et al. (2012) encontraram retrações radial e tangencial de 3,51 e 5,92 para araucária com 38 anos.

A média encontrada para o coeficiente de anisotropia, segundo a classificação de Moreschi (2010) é caracterizada como de excelente estabilidade. Para o presente trabalho o coeficiente anisotrópico não apresentou diferença estatística para as posições ao nível de 5\% de probabilidade, indicando retração homogênea entre as diferentes posições radiais das amostras, apresentando baixa tendência ao fendilhamento e empenamento.

O baixo coeficiente de anisotropia pode ser atribuído à menor densidade básica, por apresentar menor quantidade de madeira por unidade de volume, limitando seu uso para diversos fins, e por isso são necessárias medidas para atenuar o efeito anisotrópico. Entretanto, devido ao fato do material utilizado nesta pesquisa ter passado por um processo de secagem natural antes da realização do estudo, pode-se afirmar que estes resultados sofreram a influência do fenômeno da histerese.

Os valores médios foram inferiores à média relatada por outros autores para a espécie. Melo et al. (2010) obtiveram o valor médio de 1,41 para o lenho juvenil e de 1,64 para o lenho adulto. Mattos et al. (2009) em estudo realizado com madeira de araucária, encontrou valor médio de coeficiente de anisotropia de 1,41, semelhante ao encontrado neste estudo. Os autores concluíram que a estabilidade da madeira apresentou menor variação com o aumento da densidade básica quando comparada com Pinus elliottii e Pinus taeda. É importante salientar que estas variações ocorridas entre trabalhos de mesma espécie, podem ser explicadas por fatores como ritmo de crescimento, porcentagem de lenho tardio e inicial, assim como pela posição no tronco, entre outros.

No entanto, não é recomendado fazer a análise do fator anisotrópico de forma isolada para caracterizar a madeira, pois assim pode-se obter uma falsa impressão de estabilidade, o que é justificado pelos coeficientes anisotrópicos de retração baixos, oriundos de retrações tangencial e radial elevados, revelando uma madeira com alta instabilidade dimensional. Este exemplo é muito recorrente especialmente para o Eucalyptus grandis, que frequentemente apresenta valores bem baixos de coeficiente anisotrópico, mas de conhecida instabilidade dimensional (SILVA et al., 2010). Assim, o indicado é relacionar o coeficiente anisotrópico com as retrações lineares e volumétrica.

\section{Correlação de Pearson}

De acordo com a tabela 5, observou-se que a partir do coeficiente de correlação (r) que a relação da densidade básica com as demais propriedades físicas varia entre baixa e média, explicando proporção semelhante da densidade básica para cada uma das características.

Oliveira, Tomazello Filho e Fiedler (2010) julgam ser errôneo generalizar a relação entre retratibilidade e densidade básica da madeira, podendo ocorrer que em madeiras densas, a presença de elevados teores de extrativos hidrófobos nas paredes celulares pode resultar em baixa retração volumétrica. Para Glass e Zelinka (2010) a contração está associada à densidade básica da madeira, entretanto, o tamanho e a geometria da madeira, assim como a velocidade de secagem também podem influenciar na retração.

FLORESTA, Curitiba, PR, v. 47, n. 3, p. 333 - 341, jul. / set. 2017

Dias, A. C. C. et al.

ISSN eletrônico 1982-4688

DOI: $10.5380 /$ rf.v47i1.50732 
Resultado similar a este estudo foi obtido por Mattos et al. (2009) e segundo estes autores, a equação de regressão da contração volumétrica em função da densidade básica apresentou coeficiente de determinação igual a 0,07 e que, na maior parte das árvores (ou idades), a densidade básica explica pouco mais de $40 \%$ somando as contrações radial e tangencial. Conclusões opostas foram obtidas por Melo et al. (2010) ao afirmar que, as densidades obtiveram correlações significativas com todos os coeficientes, exceto com o fator anisotrópico.

Deste modo, notamos que apenas a densidade não explica os valores médios encontrados para as propriedades avaliadas, demonstrando o quanto as propriedades físico-químicas e anatômicas estão interligadas. Assim, compreende-se a importância de relacionar o maior número de parâmetros avaliados quando se deseja avaliar a qualidade da madeira, inclusive àquelas destinadas para painéis laminados, pois, apenas a densidade não é capaz de inferir se a madeira apresentará boa estabilidade dimensional e ausência de colapso.

\section{CONCLUSÃO}

- Portanto, pode-se afirmar que a espécie Araucária angustifolia possui boa característica de forma do fuste segundo a classificação de IBDF (1984), pois apresenta baixa conicidade e achatamento acarretando em boa qualidade das lâminas, por apresentarem baixo crescimento excêntrico da medula e maior homogeneidade da densidade, tendendo a apresentar rendimento satisfatório devido suas características morfométricas.

- A variação no sentido longitudinal é expressiva e afeta a qualidade da madeira quando se deseja matéria prima para laminação, e para reduzir a heterogeneidade ao longo do fuste, o recomendado é dividir em toras de menor comprimento a fim de reduzir a variação do mesmo.

- Os valores encontrados para a densidade no sentido radial caracterizaram a madeira como de baixa densidade, definindo-a como de baixa propensão a empenamentos, rachaduras e colapso. No entanto, apresenta variação da medula para a casca, podendo ser destinada a painéis laminados, mas tendo o cuidado quanto a sua heterogeneidade neste sentido.

- Por se tratar de uma madeira que apresentou baixos valores de retração volumétrica e coeficiente anisotrópico, a araucária possui alta estabilidade dimensional, desta forma acarretará em poucos defeitos durante a secagem. Este fator é positivo para qualidade em laminação, o que justifica a ampla utilização desta espécie;

- A densidade básica apresentou baixa relação com as propriedades físicas, demonstrando que apenas a densidade não é indicativo para determinar a qualidade final do produto.

\section{AGRADECIMENTO}

À empresa Palmasola pela concessão do material para realização deste estudo.

\section{REFERENCIAS}

AMORIN, P. G. R.; GONÇALVEZ, J. C.; CAMARGOS J. A. A. Propriedades da madeira de Pinus caribaea e Eucalyptus grandis estimadas por colorimetria. Revista Cerne, Lavras, v. 19, n. 3, p. 461-466, 2013.

ASSOCIAÇÃO BRASILEIRA DE PRODUTORES DE FLORESTAS PLANTADAS (ABRAF). Anuário estatístico da ABRAF 2013 ano base 2012. Brasília, DF, 2013. 146 p.

ASSOCIAÇÃO BRASILEIRA DE NORMAS TÉCNICAS. NBR 7190: Projetos de estruturas de madeira. Rio de Janeiro, 1997. 107p.

ASSOCIAÇÃO BRASILEIRA DE NORMAS TÉCNICAS. NBR 11941: Madeira: Determinação da densidade básica. Rio de Janeiro. 2003, 6p.

CURTO, R. A.; MATTOS, P. P.; BRAZ, E. M.; NETTO, S. P.; ZACHOW, R. Density of Araucaria angustifolia wood from overstocked stand. Pesquisa Florestal Brasileira, Colombo, v. 36, n. 85, p. 51-59, 2016.

INSTITUTO BRASILEIRO DO MEIO AMBIENTE E DOS RECURSOS NATURAIS RENOVÁVEIS IBAMA. Banco de dados de madeiras brasileiras. Disponível em: 〈http://www.ibama.gov.br >. Acesso em: 30 dez. 2015.

INSTITUTO BRASILEIRO DE DESENVOLVIMENTO FLORESTAL. Norma para medição e classificação de toras de madeiras de folhosas. Brasília: brasiliana, 1984. 42p. 
FIGUEREDO FILHO, A. KOHLER, S. V.; FELDE, J. L.; DIAS, A. N. Dinâmica do afilamento do tronco e da produção de madeira em plantios de Araucaria angustifolia. Cerne, Lavras, v. 20 n. 4, p. 595-603, 2014.

FIGUEIRDO FILHO, A.; RETSLAFF, F. A. S.; KOHLER, S. V.; BECKER, M.; BRANDES, D. Efeito da Idade no Afilamento e Sortimento em Povoamentos de Araucaria angustifolia. Floresta e Ambiente, Rio de Janeiro, v. 22, n. 1, p. 50-59, 2015.

GLASS, S. V.; ZELINKA, S. L. Moisture Relations and Physical Properties of Wood. Wood handbook.Wood as an engineering material. Madison, WI. Chapter, v. 4, 2010. 508p.

HILLIG, É. MACHADO, G. O.; HOLK, D. L.; CORRADI, G. M. Propriedades físicas da madeira de Araucaria angustifolia (Bert.) O. Kuntze em função da posição no fuste para diferentes idades. Cerne, Lavras, v. 18, n. 2, p. $257-263,2012$

INSTITUTO BRASILEIRO DE DESENVOLVMENTO FLORESTAL. Norma para medição e classificação de toras de madeiras de folhosas. Brasília: Brasiliana, 1984. 42p.

KLITZKE, R. J. Secagem da madeira. In: José Tarcísio da Silva Oliveira, Nilton César Fiedler, Marcelo Nogueira. (Org.). Tecnologias aplicadas ao setor madeireiro. Visconde do Rio Branco: Suprema Gráfica e Editora Ltda, 2007, v.1, p. 271-366.

MATTOS, P. P.; BORTOLI, C.; MARCHESAN, R.; ROSOT, N. C. Caracterização física, química e anatômica da madeira de Araucaria angustifolia (Bert.) O. Kuntze. Colombo, Embrapa Floresta 2006. (Comunicado Técnico, 160).

MATTOS, J. R. O pinheiro brasileiro. Florianópolis: Ed. da UFSC, 2011. 700p.

MELO, R. R.; STANGERLIN, D. M.; DEL MENEZZI, C. H. S.; GATTO, D. A.; CALEGARI, L. Caracterização física e mecânica da madeira de Araucaria angustifolia (Bertol.) Kuntze. Revista brasileira de ciências agrárias, Recife, v. 5, n. 1, p. 67-73, 2010.

MORESCHI, J. C. Propriedades Tecnológicas da Madeira. $3^{\circ}$ edição. Departamento de Engenharia e Tecnologia Florestal da UTFPR. 177p. 2010.

OLIVEIRA, R. M. Wettability, Shrinkage and Color Changes of Araucaria angustifolia After Heating Treatment. Materials Research. São Carlos, v. 13, n. 3, p. 351-354, 2010.

REITZ, R.; KLEIN, R. M. Araucariaceae, Itajaí: Herbário Barbosa Rodrigues, 1966. 29p.

ROSA, T. H.; HILLIG, E.; VIANA, L. C. Propriedades físicas da madeira de Araucaria angustifolia (bert.) o. Kuntze e sua relação com os anéis de crescimento.Ciência da Madeira, Pelotas, v. 04, n. 01, p. 01-14, 2013.

SANQUETTA, C. R.; DOLCI, M.; DALLA CORTE, A. P.; SANQUETTA, M. N. I.; PELISSARI, A. L. Estimação de volumes de Araucaria angustifolia (bertol.) o. kuntze por fatores de forma em classes diamétricas e modelos de regressão. Enciclopédia biosfera, Goiânia, v. 13, n. 23, p. 2016.

SILVA OLIVEIRA, J. T.; FILHO, M. T.; FIEDLER, N. C. Avaliação da retratibilidade da madeira de sete espécies de Eucalyptus. Revista Árvore, Viçosa, v. 34, n. 5, p. 929-936, 2010.

VALÉRIO, A. F.; WATZLAWICK, L. F.; SANTOS, R. T.; BRANDELERO, C.; KOEHLER, H. S. Quantificação de resíduos e rendimento no desdobro de Araucaria angustifolia (Bert.) O. Kuntze. Revista Floresta, Curitiba, v. 37, p. 387-398, 2007.

WEHR, N. J.; TOMAZELLO FILHO, M. Caracterização dos anéis de crescimento de árvores de Araucaria angustifolia (Bert.) O. Ktze, através da microdensitometria de raio X. Scientia Florestalis. Piracicaba, n. 58, p. 161-170, (2000).

WELLING, J. European Drying group - Recommendation. Assessment of drying quality of timber. Piloredition. 1994. 30p. 